\title{
Dual-modality drainage of infected and symptomatic walled-off pancreatic necrosis: Long-term clinical outcomes
}

\section{Andrew S. Ross, Shayan Irani, S. Ian Gan, Flavio Rocha, Justin Siegal, Mehran Fotoohi, Ellen Hauptmann, David Robinson, Robert Crane, Richard Kozarek, Michael Gluck}

Gastrointestinal Endoscopy 2014;79:929-35

The treatment of pancreatic necrosis has evolved over a period from surgical necrosectomy to minimally invasive surgical, endoscopic, and percutaneous techniques. ${ }^{[1-4]}$ The percutaneous drainage (PCD) of pancreatic necrosis has been shown to be an effective primary approach as well as a step up approach with good results. ${ }^{[5,6]}$ In spite of these good results and avoidance of surgery in the majority of patients, an increased risk of development of chronic pancreaticocutaneous fistula (PCF) following PCD due to the presence of disconnected pancreatic duct syndrome (DPDS) is an important concern. ${ }^{[7]}$

To circumvent this risk of PCF, the authors of the current study had developed a combined endoscopic and percutaneous approach to symptomatic walled off pancreatic necrosis (WOPN). In this dual-modality drainage (DMD) transenteric stents are placed endoscopically into the WOPN immediately after PCD ${ }^{[8]}$ This allows redirection of pancreatic juice into the gastrointestinal tract and, therefore, decreasing the risk of PCF formation. The authors had initially described the short-term results of this technique in 15 patients and in this current study the authors describe the long-term results in 117 patients of WOPN.

Over 6 years, 100 and 17 patients with symptomatic WOPN (65\%; 74 men; mean age 55.2 years) were treated with DMD. Fifty-five patients underwent DMD for infected necrosis whereas the remaining 62 patients were treated for failure to clinically improve or having gastric outlet obstructive symptoms. The data of these patients were collected prospectively and thereafter reviewed for the current study. The WOPN was initially accessed under ultrasound and/or computed tomography (CT) guidance. The path of the PCD was kept relatively straight to avoid kinks with patient motion, and the catheter tip was directed toward the dependent portion of the collection so that gravity assisted drainage could be achieved. Following placement of PCD, the patient was given general anesthesia, and endoscopic drainage of WOPN was performed. Two $7 \mathrm{~F}$ (varying lengths) double pigtail stents (Wilson Cook) were placed across the gastric or duodenal wall to maintain the tract. In the case of multiple WOPN, multiple PCD catheters were placed. Once placed, transenteric stents were not intentionally manipulated throughout the treatment course. A nasoenteric tube was placed depending upon the nutritional status of the patient and antibiotics were prescribed as per the culture report. The follow-up imaging was performed as per the patient's clinical condition. The PCD were periodically up-sized so as to allow adequate drainage of necrotic debris with maximum caliber of $24 \mathrm{~F}$. Drains were flushed with $10-20 \mathrm{~mL}$ of saline solution 3 times daily until the collections had completely resolved on CT scan. Once WOPN resolved, the drain was capped, and a repeat $\mathrm{CT}$ abdomen was performed 2 weeks later to look for recurrence of WOPN. If there was no residual fluid collection, the PCD were removed.

Pancreatography was obtained by magnetic resonance imaging or endoscopic retrograde cholangiopancreatography (ERCP), and the integrity of main pancreatic duct (PD) was used to determine the duration of transenteric drain placement. In patients with DPDS, transenteric stents were left in place indefinitely whereas, in those patients whose stents migrated spontaneously, replacement was not performed unless clinically indicated. In those with intact pancreatic ducts, transenteric stents were removed endoscopically (unless spontaneous migration had occurred). Transpapillary stents were placed at the discretion of the treating endoscopist.

Of 117 patients, 103 (88\%) completed treatment, with all percutaneous drains removed, 10 (8.5\%) are still undergoing treatment, and 4 patients (3.4\%) died with PCD in place. The mean CT severity index of the entire cohort was 7.8 with 75 patients (64.1\%) having DPDS. The mean time interval from onset of severe acute pancreatitis to initiation of DMD was 75.5 (82.2) days. The median duration of PCD was 63 days with this group of patients undergoing a mean of 7 (3.3) CT scans and 6.1 (3.0) interventional radiology-directed PCD catheter investigations and none of these patients developed PCF. Transpapillary PD stents were placed in 53 patients (45.2\%). The median duration of follow-up was 749.5 days of the patients required surgery.

The complications of DMD included self-limited bleeding in 4 patients, asymptomatic pneumoretroperitoneum in 1 patient and infection of the pancreatic bed due to premature removal of the PCD catheter and none required surgery. The late complications included contained colonic perforation because of spontaneous migration of the transgastric stents. Three patients required surgery after completion 
of DMD: 2 patients underwent distal pancreatectomy for ongoing pain, 1 patient underwent a gastrojejunostomy for gastric outlet obstruction. There were 4 deaths: 1 patient died of multiple system organ failure (found to have occult pancreatic cancer at autopsy), 2 patients died from persistent hemorrhage from the pancreatic bed, and 1 patient died from a mucous plug while in a rehabilitation facility after discharge.

Eight (7.8\%) patients developed recurrent PFCs, and four of these collections were seen in patients with DPDS due to spontaneous migration of the transenteric stents, and 4 were seen in patients with an intact pancreas. Three of the patients were managed conservatively for asymptomatic fluid collections, and cystoduodenostomy with resolution of symptoms was performed in the fourth patient. In 2 of the 4 patients with intact pancreas, the recurrent PCFs were related to high-grade stenosis of the PD and these were initially managed with transpapillary PD stent placement. However, the collections recurred after stent removal but as these were asymptomatic, these collections were managed conservatively. The other 2 patients developed peripancreatic abscesses that were successfully managed with PCD. The authors concluded that DMD for symptomatic and infected WOPN results in favorable clinical outcomes with complete avoidance of PCF, surgical necrosectomy, and major procedure-related adverse events.

\section{Commentary}

With the advent of minimally invasive treatment modalities for WOPN, a number of techniques using endoscopic drainage alone or combination of endoscopic and PCD have been described with varying results. The complete removal of the necrotic tissue is the ultimate goal all the treatment modalities. The advantage of combining endoscopic drainage with PCD is the creation of internal drainage route for pancreatic secretions, and this will be helpful in patients with DPDS by avoiding the formation of PCF. The other advantage of this combination could be decreased frequency of endoscopy-related complications as compared to patients undergoing endoscopic drainage alone or direct endoscopic necrosectomy due to smaller caliber of balloon dilation performed during DMD (only up to $8 \mathrm{~mm}$ vs. $15 \mathrm{~mm}$ or higher with direct endoscopic necrosectomy), lack of advancement of the endoscope into the cavity and use of PCD catheters for debridement as opposed to endoscopic accessories that may require cautery. However, a major drawback of this approach is the presence of PCD catheter for a median duration of 60 days. Such a long duration of PCD draining protein-rich fluid is a disadvantage and also associated with risk of malnutrition. The results of this study also confirm our earlier results that had shown the safety and efficacy of leaving transmural stents indefinitely in patients with DPDS. ${ }^{[7]}$ This study adds on to the data that has shown that minimally invasive approach is safe and effective for the treatment of WOPN.

\section{Transpapillary dilation of refractory severe biliary stricture or main pancreatic duct by using a wire-guided diathermic dilator (with video)}

\begin{abstract}
Hiroshi Kawakami, Masaki Kuwatani, Kazumichi Kawakubo, Kazunori Eło, Shin Haba, Taiki Kudo, Yoko Abe, Shuhei Kawahata, Naoya Sakamoto
\end{abstract}

Gastrointestinal Endoscopy 2014;79:338-343

Endoscopic transpapillary stent placement has been shown to be an effective therapeutic modality for the treatment of benign and malignant biliary duct (BD) and PD strictures. ${ }^{[9,10]}$ The stents are usually placed after bougie or balloon dilatation of these ductal strictures. In some cases, the strictures are very tight, and only guide wire can be taken across these strictures. These refractory strictures have been usually dilated using screw drill like Soehendra extractor that is pushed over the guide wire. ${ }^{[1]]}$ Other methods that have been reported for treating these tight and refractory biliary strictures include dissection of difficult PD strictures using a needle-knife or wire-guided snare forceps. ${ }^{[12-14]}$

Diathermic dilators that are used in the endoscopic management of pancreatic fluid collections have been used for creating and enlarging the cystoenterostomy tract. ${ }^{[15]}$ The authors of the current study had earlier reported a case of where they used this diathermic dilator to treat tight $\mathrm{BD}$ strictures. ${ }^{[16]}$ In this prospective observational cohort study, the authors evaluated the safety and efficacy of wire-guided diathermic dilator in patients with severe and tight $\mathrm{BD}$ and $\mathrm{PD}$ strictures.

Twenty-two consecutive patients (12 55\%) males; mean age 62 years (range, $32-80 ; \mathrm{BD}$ stricture 16 patients and $\mathrm{PD}$ stricture in 6 patients) who were candidates for $\mathrm{BD} / \mathrm{PD}$ decompression were treated with a wire-guided diathermic dilator. Patients with $<20$ years of age, performance status value $>3$ according to the Eastern Cooperative Oncology Group scale, mental disability, contrast medium allergy, severe heart disease (New York Heart Association class III or IV heart failure), severe pulmonary disease (peripheral oxygen saturation $<90 \%$ ), ampullary neoplasm, infiltration of the papillary area by pancreatic cancer, acute pancreatitis, chronic pancreatitis with acute exacerbation at time of the ERCP, Roux-en-Y surgery, pregnancy, women wishing to become pregnant, nursing mothers, and patients refusing consent were excluded from the study. 